\title{
Pegfilgrastim on febrile neutropenia in pediatric and adolescent cancer patients: A systematic review and single-arm meta-analysis
}

\author{
Xia Zhu ${ }^{1}$, Weiling Zhang ${ }^{1}$, Yi Zhang ${ }^{1}$, Yizhuo Wang ${ }^{1}$, Hui-Min $\mathrm{Hu}^{1}$, and Dongsheng \\ Huang ${ }^{1}$ \\ ${ }^{1}$ Beijing Tongren Hospital
}

August 28, 2020

\begin{abstract}
Background: Granulocyte colony-stimulating factors (G-CSF) can be used as prophylaxis for febrile neutropenia (FN) in adults. There is no meta-analysis about the effects of pegfilgrastim on the occurrence of FN in pediatric/adolescent cancer patients. Purpose: To determine the effect of pegfilgrastim on FN in pediatric and adolescent cancer patients. Methods: PubMed, Embase, and the Cochrane library were searched for studies published prior to April 7, 2020. The outcome was the occurrence of FN. For analyses showing high heterogeneity (I2>50\% and Q-test $\mathrm{P}<0.10$ ), the random-effect model was used; otherwise, the fixed-effect model was used. Results: Eight studies were included, comprising 167 patients and 550 courses of treatment. Pegfilgrastim decreased the rate of FN compared with controls $(\mathrm{ES}=0.26,95 \% \mathrm{CI}$ : 0.15-0.36, $\mathrm{P}<0.001)$; this was observed in prospective and retrospective studies. There was no difference between pegfilgrastim and filgrastim for the rate of FN in children receiving chemotherapy $(\mathrm{OR}=0.68,95 \% \mathrm{CI}: 0.20-2.23, \mathrm{P}=0.520)$. Pegfilgrastim decreased the rate of grade $4 \mathrm{FN}$ compared with controls ( $\mathrm{ES}=0.40,95 \% \mathrm{CI}$ : 0.16-0.63, $\mathrm{P}=0.017$ ); this was observed when analyzing the courses of treatment, but not the patients. Pegfilgrastim had no impact on treatment delays due to $\mathrm{FN}(\mathrm{ES}=0.05,95 \% \mathrm{CI}$ : $-0.00-0.10, \mathrm{P}=0.061)$. Pegfilgrastim decreased the rate of severe neutropenia compared with controls $(\mathrm{ES}=0.39,95 \% \mathrm{CI}$ : 0.04-0.74, $\mathrm{P}=0.031$ ). Conclusion: Compared with the control, pegfilgrastim significantly decreases the occurrence of $\mathrm{FN}$, grade $4 \mathrm{FN}$, and severe neutropenia in pediatric patients receiving chemotherapy. Pegfilgrastim had no impact on treatment delays due to FN.
\end{abstract}

\section{Introduction}

Severe neutropenia (SN) is defined as a current or anticipated absolute neutrophil count $(\mathrm{ANC})<500$ cells $/ \mathrm{mm}^{3}{ }^{1-3}$ Febrile neutropenia $(\mathrm{FN})$ is defined as a single fever $\left(38.3^{\circ} \mathrm{C}\right)$ or sustained elevated temperature $\left(38^{\circ} \mathrm{C}\right)$ in a patient with a current or anticipated absolute neutrophil count $\left(\right.$ ANC) $<500$ cells $/ \mathrm{mm}^{3} .{ }^{1-3}$ FN most commonly occurs in patients receiving cytotoxic chemotherapy and affects $10 \%-50 \%$ of patients with solid tumor malignancies and $>80 \%$ of patients with hematologic malignancies. The complications are delayed treatments, dose reduction, and increased risk of infection. ${ }^{1-3}$ Most infections are believed to arise from the patient's endogenous flora, with identified sites including bacteremia in 20\%-35\% and respiratory, urinary, gastrointestinal (GI), or skin infections in an additional 20\%-30\% ${ }^{1-3}$ High-risk FN is defined as anticipated prolonged ( $>7$ days duration) and profound neutropenia (ANC [?]100 cells/ $\mathrm{mm}^{3}$ following cytotoxic chemotherapy) and is associated with significant medical comorbid conditions, including hypotension, pneumonia, new-onset abdominal pain, or neurologic changes; low-risk FN is defined by anticipated brief neutropenic periods ([?]7 days) and no or few comorbidities. ${ }^{3-5}$

The current guidelines recommend granulocyte colony-stimulating factors (G-CSF) for primary prophylaxis of chemotherapy-induced FN. ${ }^{3,6}$ The majority of available G-CSF treatments are short-acting, but new 
long-acting agents (eg, pegfilgrastim and lipegfilgrastim) are being made available. Pegfilgrastim is the most widely approved long-acting G-CSF available worldwide. ${ }^{7,8}$ Prophylaxis with G-CSF has been shown to reduce the incidence of $\mathrm{FN}$ and to improve the outcomes of cancer treatment. ${ }^{9,10}$ Unfortunately, the use of G-CSF in clinical practice is suboptimal. ${ }^{11-14}$ Additional studies indicating the usefulness of G-CSF might improve this outcome.

In clinical trials, pegfilgrastim has been shown to have a favorable efficacy and safety profile. ${ }^{15-19} \mathrm{~A}$ metaanalysis showed that G-CSF in adult patients receiving chemotherapy is effective and safe, including pegfilgrastim and biosimilars. ${ }^{20}$ Another meta-analysis of pegfilgrastim showed that it reduced the occurrence of FN compared with filgrastim. ${ }^{21}$ The meta-analysis by Cornes et al. ${ }^{22}$ showed better outcomes with longacting G-CSF compared with short-acting ones. The use of filgrastim or pegfilgrastim vs. placebo improved the overall survival (OS) of patients receiving chemotherapy. ${ }^{23}$

Nevertheless, there is only one guideline for pediatric and adolescent cancer patients regarding the use of $\mathrm{G}-\mathrm{CSF},{ }^{4}$ and there is no meta-analysis about the effects of pegfilgrastim on the occurrence of FN in pediatric and adolescent cancer patients. Therefore the purpose of this meta-analysis was to determine the effect of pegfilgrastim on FN in pediatric and adolescent cancer patients.

\section{Methods}

\section{Literature review}

This meta-analysis was performed in accordance with the Preferred Reporting Items for Systematic Reviews and Meta-Analyses (PRISMA) reporting guidelines. ${ }^{24}$ Since no original clinical raw data was collected or used, ethical approval was not requested for this meta-analysis.

PubMed, Embase, and the Cochrane library were searched for studies published prior to April 7, 2020, using the MeSH terms of "child", "pediatrics", "adolescent", and "pegfilgrastim", combined with relevant key words. The eligibility criteria were: 1) patients: pediatric or adolescent cancer patients receiving chemotherapy; 2) intervention: pegfilgrastim; 3) outcome: febrile neutropenia, neutropenia or treatment delay; and 4) full text published in English.

\section{Data extraction and quality assessment}

Potentially relevant publications were screened and evaluated by two reviewers (*Zhang Weiling* and *Zhang $\mathrm{Yi}^{*}$ ) double-blindly, with a third reviewer ( ${ }^{*} \mathrm{Hu}$ Huimin*) being requested to solve any disagreement. A structured data collection form was developed. Two researchers (**Zhu Xia* and *Wang Yizhuo*) independently extracted the data, including authors, year of publication, country, study design, sample size, age, percentage of males, episodes or courses, disease, dosage, etc.

The randomized controlled trials (RCTs) were evaluated using the Cochrane risk bias tool. ${ }^{25,26}$ Observational studies were evaluated according to the Newcastle-Ottawa scale (NOS). ${ }^{27}$ Case series studies were evaluated according to the National Institute for Health and Clinical Excellence (NICE) method. ${ }^{28}$ Nonrandomized trials were evaluated according to the revised and validated version of the methodological index for non-randomized studies (MINORS). ${ }^{29}$ Crossover studies were evaluated according to Hong Ding's quality assessment standard ${ }^{30}$.

\section{Statistical analysis}

All analyses were performed using STATA MP 14.0 (StataCorp, College Station, Texas, USA). Effect size (ES) and odds ratio (OR) and their 95\% confidence intervals (CI) were used for analysis. Statistical heterogeneity among the studies was calculated using Cochran's Q-test and the $\mathrm{I}^{2}$ index. An $\mathrm{I}^{2}>50 \%$ and Q-test 
$\mathrm{P}<0.10$ indicated high heterogeneity, and the random-effects model was used when high heterogeneity was present among studies; otherwise, the fixed-effects model was applied. P-values $<0.05$ were considered statistically different. Potential publication bias, Egger's test, and Begg's test were not performed due to the small number of studies included in each analysis. ${ }^{25}$

\section{RESULTS}

\section{Study selection}

Supplementary Figure 2 presents the study selection process. A total of 369 records were identified, and 303 were left after removing the duplicates. Then, 226 were excluded after screening, and 77 full-text papers were assessed for eligibility. Among them, 69 were excluded because of study aim/design $(n=25)$, population $(\mathrm{n}=33)$, exposures $(\mathrm{n}=6)$, data previously analyzed $(\mathrm{n}=1)$, and non-English text $(\mathrm{n}=4)$.

Finally, eight studies were included. There were four prospective studies ${ }^{31-34}$ and four retrospective studies (Supplementary Table 1). ${ }^{35-38}$ There were a total of 167 patients and 550 episodes/courses. Four studies had no control group, and four studies used filgrastim as control. Supplementary Table 2 presents the quality evaluation of the included study.

\section{Rate of FN}

Six studies were included to determine the rate of $\mathrm{FN}^{31,32,35-38}$ The results showed that pegfilgrastim decreased the rate of $\mathrm{FN}$ compared with controls ( $\mathrm{ES}=0.26,95 \% \mathrm{CI}: 0.15-0.36, \mathrm{P}<0.001 ; \mathrm{I}^{2}=88.0 \%$, $\left.\mathrm{P}_{\text {heterogeneity }}<0.001\right)$ (Figure 1 and Table 1 ). The effect was observed in prospective (ES=0.23, 95\%CI: 0.12$\left.0.33, \mathrm{P}<0.001 ; \mathrm{I}^{2}=0.0 \% \%, \mathrm{P}_{\text {heterogeneity }}=0.834\right)^{31,32}$ and retrospective $(\mathrm{ES}=0.27,95 \% \mathrm{CI}: 0.13-0.41, \mathrm{P}<0.001$; $\left.\mathrm{I}^{2}=92.7 \%, \mathrm{P}_{\text {heterogeneity }}<0.001\right)^{35-38}$ studies.

\section{Rate of FN for pegfilgrastim vs. filgrastim}

Three studies compared pegfilgrastim vs. filgrastim for the occurrence of FN. ${ }^{32,36,38}$ The results showed that there was no difference between pegfilgrastim and filgrastim for the rate of $\mathrm{FN}$ in children receiving chemotherapy $\left(\mathrm{OR}=0.68,95 \% \mathrm{CI}: 0.20-2.23, \mathrm{P}=0.520 ; \mathrm{I}^{2}=80.3 \%, \mathrm{P}_{\text {heterogenity }}=0.006\right)$ (Figure 2 and Table 1).

\section{Rate of grade $4 \mathrm{FN}$}

Four studies analyzed the occurrence of grade $4 \mathrm{FN}^{31,34-36}$ The results showed that pegfilgrastim decreased the rate of grade $4 \mathrm{FN}$ compared with controls $\left(\mathrm{ES}=0.40,95 \% \mathrm{CI}: 0.16-0.63, \mathrm{P}=0.017 ; \mathrm{I}^{2}=96.2 \%\right.$, $\left.\mathrm{P}_{\text {heterogenity }}<0.001\right)$ (Figure 3 and Table 1). Similar results were observed when considering the courses of treatment ( $\mathrm{ES}=0.36,95 \% \mathrm{CI}$ : 0.06-0.66, $\left.\mathrm{P}=0.001 ; \mathrm{I}^{2}=97.3 \%, \mathrm{P}_{\text {heterogenity }}<0.001\right),{ }^{31,35,36}$ but not when considering the patients ( $\left.\mathrm{ES}=0.45,95 \% \mathrm{CI}:-0.12-1.03, \mathrm{P}=0.123 ; \mathrm{I}^{2}=97.0 \%, \mathrm{P}_{\text {heterogenity }}<0.001\right)$.

\section{Rate of SN}

Two studies analyzed the occurrence of SN. ${ }^{36,37}$ The results showed that pegfilgrastim decreased the rate of SN compared with controls ( $\mathrm{ES}=0.39,95 \% \mathrm{CI}$ : 0.04-0.74, $\mathrm{P}=0.031 ; \mathrm{I}^{2}=98.0 \%, \mathrm{P}_{\text {heterogenity }}<0.001$ ) (Figure 4 and Table1).

Treatment delays 
Three studies examined the treatment delays due to FN. ${ }^{31,35,37}$ Pegfilgrastim had no impact on treatment delays due to $\mathrm{FN}\left(\mathrm{ES}=0.05,95 \% \mathrm{CI}\right.$ : $-0.00-0.10, \mathrm{P}=0.061 ; \mathrm{I}^{2}=77.8 \%$, $\left.\mathrm{P}_{\text {heterogenity }}=0.011\right)$ (Figure 5 andTable $1)$.

\section{Sensitivity analysis}

When considering the occurrence of $\mathrm{FN}$ for pegfilgrastim vs. filgrastim, no single study influenced the results (Supplementary Figure S1).

\section{Discussion}

G-CSF can be used as prophylaxis for $\mathrm{FN}^{3,6}$ but there is no meta-analysis about the effects of pegfilgrastim on the occurrence of FN in pediatric/adolescent cancer patients. Therefore, this study aimed to determine the effect of pegfilgrastim on $\mathrm{FN}$ in pediatric and adolescent cancer patients. The results suggest that compared with the control, pegfilgrastim significantly decreases the occurrence of FN, grade $4 \mathrm{FN}$, and severe neutropenia in pediatric patients receiving chemotherapy. Pegfilgrastim had no impact on treatment delays due to FN.

FN-related mortality represents an important burden for cancer patients and i this why FN was selected as the primary outcome in the present study. ${ }^{3}$ FN prophylaxis using G-CSF is a well-recognized strategy in adult patients with cancer, as recommended by guidelines. ${ }^{3,6}$ In adults, studies showed that long-acting G-CSF achieves better outcomes than short-acting G-CSF, ${ }^{15-19}$ and meta-analyses in adults also indicated the superiority of long-acting G-CSF. ${ }^{20-23}$ Nevertheless, only one guideline ${ }^{4}$ but no meta-analysis is currently available specifically for the use of G-CSF in pediatric patients, and the guidelines highlight the need for additional evidence. ${ }^{4}$ The present meta-analysis is the first to examine the rate of FN when using pegfilgrastim, as well as the rates of SN and grade $4 \mathrm{FN}$. Those results in children and adolescents are supported by the results in adults. ${ }^{20-23}$ Nevertheless, analyses in adults showed that pegfilgrastim prevented treatment delays and dose reductions ${ }^{20-23}$ since the neutrophil values have a higher likelihood to be within the adequate ranges, but this was not observed in the present meta-analysis in pediatric populations. This is surprising since chemotherapy-induced SN and FN are considered as the first reason for dose-limiting toxicity in pediatric cancer, ${ }^{31}$ but it has been reported that only a small numbers of cycles were started later in pediatric patients treated with G-CSF. ${ }^{31,35}$ A previous meta-analysis showed that filgrastim reduced the rate of FN by $20 \%$ and shortened hospitalization time, but with no impact on infection-related mortality. ${ }^{39}$ The lack of difference in the present study between pegfilgrastim and the control arm could be because the control arm included both no use of G-CSF and filgrastim, which might have diluted the difference between the two arms. In addition, the effect of G-SCF is to stimulate the hematopoietic cells to produce more neutrophils. The hematopoietic system in children is more active than the adult counterpart and the composition in hematopoietic stem cells and their location in the body are also different. ${ }^{40,41}$ Therefore, future studies should be designed to directly compare the effects of G-CSF between children and adults. Furthermore, different chemotherapy drugs have different myelosuppression potentials, ${ }^{42,43}$ but this could not be taken into account in the present meta-analysis because of the small number of included studies.

The conclusions of this meta-analysis must be considered within its limitations. The number of studies that could be included was small, leading to a small number of patients and courses of treatment. Because of this, no selection was based on the type of cancer or the type of chemotherapy, introducing bias sue to the different aggressiveness of the diseases and treatments. If a control group was included, the control group was either filgrastim or nothing. The studies were conducted in various countries with different guidelines for the use of G-CSF. These reasons led to high heterogeneity for all analyses. Furthermore, the safety issues could not be analyzed because of the too important differences in safety outcomes among the included studies. In addition, future studies should examine the effect of pegfilgrastim biosimilars in pediatric patients with cancer. 
In conclusion, the results suggest that compared with the control, pegfilgrastim significantly decreases the occurrence of FN, grade $4 \mathrm{FN}$, and severe neutropenia in pediatric patients receiving chemotherapy. Pegfilgrastim had no impact on treatment delays due to FN.

\section{References}

1. Taplitz RA, Kennedy EB, Bow EJ, et al. Outpatient Management of Fever and Neutropenia in Adults Treated for Malignancy: American Society of Clinical Oncology and Infectious Diseases Society of America Clinical Practice Guideline Update. J Clin Oncol. 2018;36(14):1443-1453.

2. Wang XJ, Chan A. Optimizing Symptoms and Management of Febrile Neutropenia among Cancer Patients: Current Status and Future Directions. Curr Oncol Rep. 2017;19(3):20.

3. Freifeld AG, Bow EJ, Sepkowitz KA, et al. Clinical practice guideline for the use of antimicrobial agents in neutropenic patients with cancer: 2010 update by the infectious diseases society of america. Clin Infect Dis. 2011;52(4):e56-93.

4. Lehrnbecher T, Robinson P, Fisher B, et al. Guideline for the Management of Fever and Neutropenia in Children With Cancer and Hematopoietic Stem-Cell Transplantation Recipients: 2017 Update. J Clin Oncol. 2017;35(18):2082-2094.

5. Ullmann AJ, Akova M, Herbrecht R, et al. ESCMID* guideline for the diagnosis and management of Candida diseases 2012: adults with haematological malignancies and after haematopoietic stem cell transplantation (HCT). Clin Microbiol Infect. 2012;18 Suppl 7:53-67.

6. Smith TJ, Bohlke K, Lyman GH, et al. Recommendations for the Use of WBC Growth Factors: American Society of Clinical Oncology Clinical Practice Guideline Update. J Clin Oncol. 2015;33(28):3199-3212.

7. Rofail P, Tadros M, Ywakim R, Tadrous M, Krug A, Cosler LE. Pegfilgrastim: a review of the pharmacoeconomics for chemotherapy-induced neutropenia. Expert Rev Pharmacoecon Outcomes Res. 2012;12(6):699-709.

8. Renwick W, Pettengell R, Green M. Use of filgrastim and pegfilgrastim to support delivery of chemotherapy: twenty years of clinical experience. BioDrugs. 2009;23(3):175-186.

9. Aapro MS, Bohlius J, Cameron DA, et al. 2010 update of EORTC guidelines for the use of granulocytecolony stimulating factor to reduce the incidence of chemotherapy-induced febrile neutropenia in adult patients with lymphoproliferative disorders and solid tumours. Eur J Cancer. 2011;47(1):8-32.

10. Leonard RC, Mansi JL, Keerie C, et al. A randomised trial of secondary prophylaxis using granulocyte colony-stimulating factor ('SPROG' trial) for maintaining dose intensity of standard adjuvant chemotherapy for breast cancer by the Anglo-Celtic Cooperative Group and NCRN. Ann Oncol. 2015;26(12):2437-2441.

11. Kelly S, Wheatley D. Prevention of febrile neutropenia: use of granulocyte colony-stimulating factors. Br J Cancer. 2009;101 Suppl 1:S6-10.

12. Barnes G, Pathak A, Schwartzberg L. G-CSF utilization rate and prescribing patterns in United States: associations between physician and patient factors and GCSF use. Cancer Med. 2014;3(6):1477-1484.

13. Link H, Nietsch J, Kerkmann M, Ortner P, Supportive Care Group of the German Cancer S. Adherence to granulocyte-colony stimulating factor (G-CSF) guidelines to reduce the incidence of febrile neutropenia after chemotherapy-a representative sample survey in Germany. Support Care Cancer. 2016;24(1):367-376.

14. Weycker D, Hackett J, Edelsberg JS, Oster G, Glass AG. Are shorter courses of filgrastim prophylaxis associated with increased risk of hospitalization? Ann Pharmacother. 2006;40(3):402-407. 
15. Amgen Ltd. Neulasta. Summary of product characteristics. https://www.ema.europa.eu/en/documents/product-information/neulasta-epar-product-information_en.pdf. Accessed May 28, 2020. 2015.

16. Vogel CL, Wojtukiewicz MZ, Carroll RR, et al. First and subsequent cycle use of pegfilgrastim prevents febrile neutropenia in patients with breast cancer: a multicenter, double-blind, placebo-controlled phase III study. J Clin Oncol. 2005;23(6):1178-1184.

17. Mey UJ, Maier A, Schmidt-Wolf IG, et al. Pegfilgrastim as hematopoietic support for dose-dense chemoimmunotherapy with R-CHOP-14 as first-line therapy in elderly patients with diffuse large B cell lymphoma. Support Care Cancer. 2007;15(7):877-884.

18. Ozer H, Mirtsching B, Rader M, et al. Neutropenic events in community practices reduced by first and subsequent cycle pegfilgrastim use. Oncologist. 2007;12(4):484-494.

19. Pro B, Fayad L, McLaughlin P, et al. Pegfilgrastim administered in a single fixed dose is effective in inducing neutrophil count recovery after paclitaxel and topotecan chemotherapy in patients with relapsed aggressive non-Hodgkin's lymphoma. Leuk Lymphoma. 2006;47(3):481-485.

20. Wang Y, Chen L, Liu F, et al. Efficacy and tolerability of granulocyte colony-stimulating factors in cancer patients after chemotherapy: A systematic review and Bayesian network meta-analysis. Sci Rep. 2019;9(1):15374.

21. Bond TC, Szabo E, Gabriel S, et al. Meta-analysis and indirect treatment comparison of lipegfilgrastim with pegfilgrastim and filgrastim for the reduction of chemotherapy-induced neutropenia-related events. J Oncol Pharm Pract. 2018;24(6):412-423.

22. Cornes P, Gascon P, Chan S, et al. Systematic Review and Meta-analysis of Short- versus Long-Acting Granulocyte Colony-Stimulating Factors for Reduction of Chemotherapy-Induced Febrile Neutropenia. Adv Ther. 2018;35(11):1816-1829.

23. Lyman GH, Reiner M, Morrow PK, Crawford J. The effect of filgrastim or pegfilgrastim on survival outcomes of patients with cancer receiving myelosuppressive chemotherapy. Ann Oncol. 2015;26(7):14521458 .

24. Selcuk AA. A Guide for Systematic Reviews: PRISMA. Turk Arch Otorhinolaryngol. 2019;57(1):57-58.

25. Higgins JPT, Thomas J, Chandler J, et al. Cochrane Handbook for Systematic Reviews of Interventions version 6.0 (updated July 2019). London: Cochrane Collaboration; 2019.

26. Higgins JP, Altman DG, Gotzsche PC, et al. The Cochrane Collaboration's tool for assessing risk of bias in randomised trials. BMJ. 2011;343:d5928.

27. Lo CK, Mertz D, Loeb M. Newcastle-Ottawa Scale: comparing reviewers' to authors' assessments. BMC Med Res Methodol. 2014;14:45.

28. National Institute for Health and Clinical Excellence. Appendix 4 Quality of case series form. https://www.nice.org.uk/guidance/cg3/documents/appendix-4-quality-of-case-series-form2. Accessed May 28,2020 .

29. Slim K, Nini E, Forestier D, Kwiatkowski F, Panis Y, Chipponi J. Methodological index for nonrandomized studies (minors): development and validation of a new instrument. ANZ J Surg. 2003;73(9):712716.

30. Ding H, Hu GL, Zheng XY, Chen Q, Threapleton DE, Zhou ZH. The method quality of cross-over studies involved in Cochrane Systematic Reviews. PLoS One. 2015;10(4):e0120519.

31. te Poele EM, Kamps WA, Tamminga RY, Leeuw JA, Postma A, de Bont ES. Pegfilgrastim in pediatric cancer patients. J Pediatr Hematol Oncol. 2005;27(11):627-629. 
32. Wendelin G, Lackner H, Schwinger W, Sovinz P, Urban C. Once-per-cycle pegfilgrastim versus daily filgrastim in pediatric patients with Ewing sarcoma. J Pediatr Hematol Oncol. 2005;27(8):449-451.

33. Yousofian S, Miri-Aliabad G, Kiumarsi A, Ramim T. Effectiveness of filgrastim and polyethylene glycolfilgrastim in the treatment of postchemotherapy neutropenia in children: Phase I clinical trial. Indian J Med Paed Oncol. 2019;40(1):101-104.

34. Spunt SL, Irving H, Frost J, et al. Phase II, randomized, open-label study of pegfilgrastim-supported VDC/IE chemotherapy in pediatric sarcoma patients. J Clin Oncol. 2010;28(8):1329-1336.

35. Andre N, Kababri ME, Bertrand P, et al. Safety and efficacy of pegfilgrastim in children with cancer receiving myelosuppressive chemotherapy. Anticancer Drugs. 2007;18(3):277-281.

36. Milano-Bausset E, Gaudart J, Rome A, et al. Retrospective comparison of neutropenia in children with Ewing sarcoma treated with chemotherapy and granulocyte colony-stimulating factor (G-CSF) or pegylated G-CSF. Clin Ther. 2009;31 Pt 2:2388-2395.

37. Borinstein SC, Pollard J, Winter L, Hawkins DS. Pegfilgrastim for prevention of chemotherapy-associated neutropenia in pediatric patients with solid tumors. Pediatr Blood Cancer. 2009;53(3):375-378.

38. Lopez-Facundo NA, Valois-Escamilla MG, Tejocote-Romero I, Rodriguez-Castillejos C, Jaimes-Garcia Y. Cost-Benefit Study of Febrile Neutropenia Prophylactic Treatment with Pegfilgrastim vs. Filgrastim in Pediatric Patients with Solid Tumors. Gaceta Mexicana dew Oncologia. 2017;16(1):31-35.

39. Sung L, Nathan PC, Lange B, Beyene J, Buchanan GR. Prophylactic granulocyte colony-stimulating factor and granulocyte-macrophage colony-stimulating factor decrease febrile neutropenia after chemotherapy in children with cancer: a meta-analysis of randomized controlled trials. J Clin Oncol. 2004;22(16):3350-3356.

40. Copley MR, Eaves CJ. Developmental changes in hematopoietic stem cell properties. Exp Mol Med. 2013;45:e55.

41. Fernandez KS, de Alarcon PA. Development of the hematopoietic system and disorders of hematopoiesis that present during infancy and early childhood. Pediatr Clin North Am. 2013;60(6):1273-1289.

42. Akashi M, Shibuya Y, Kusumoto J, et al. Myelosuppression grading of chemotherapies for hematologic malignancies to facilitate communication between medical and dental staff: lessons from two cases experienced odontogenic septicemia. BMC Oral Health. 2013;13:41.

43. Gordon MS. Myelosuppression. Current Cancer Therapeutics . London: Current Medicine Group; 2001.

TABLE 1. Results and subgroup results

SUPPLEMENTARY TABLE 1. Characteristics of the included studies

SUPPLEMENTARY TABLE 2. Quality assessment of included studies

Figure legend

FIGURE 1. Forest plot of pooled febrile neutropenia rate

FIGURE 2. Forest plot of febrile neutropenia of pegfilgrastim vs. filgrastim

FIGURE 3. Forest plot of pooled grade 4 neutropenia rate

FIGURE 4. Forest plot of pooled severe neutropenia rate

FIGURE 5. Forest plot of pooled treatment delay due to neutropenia rate

SUPPLEMENTARY FIGURE 1.Sensitivity analysis of febrile neutropenia of pegfilgrastim vs. filgrastim SUPPLEMENTARY FIGURE 2.Study selection process.

Hosted file 
Table 1.docx available at https://authorea.com/users/354430/articles/477987-pegfilgrastimon-febrile-neutropenia-in-pediatric-and-adolescent-cancer-patients-a-systematic-reviewand-single-arm-meta-analysis

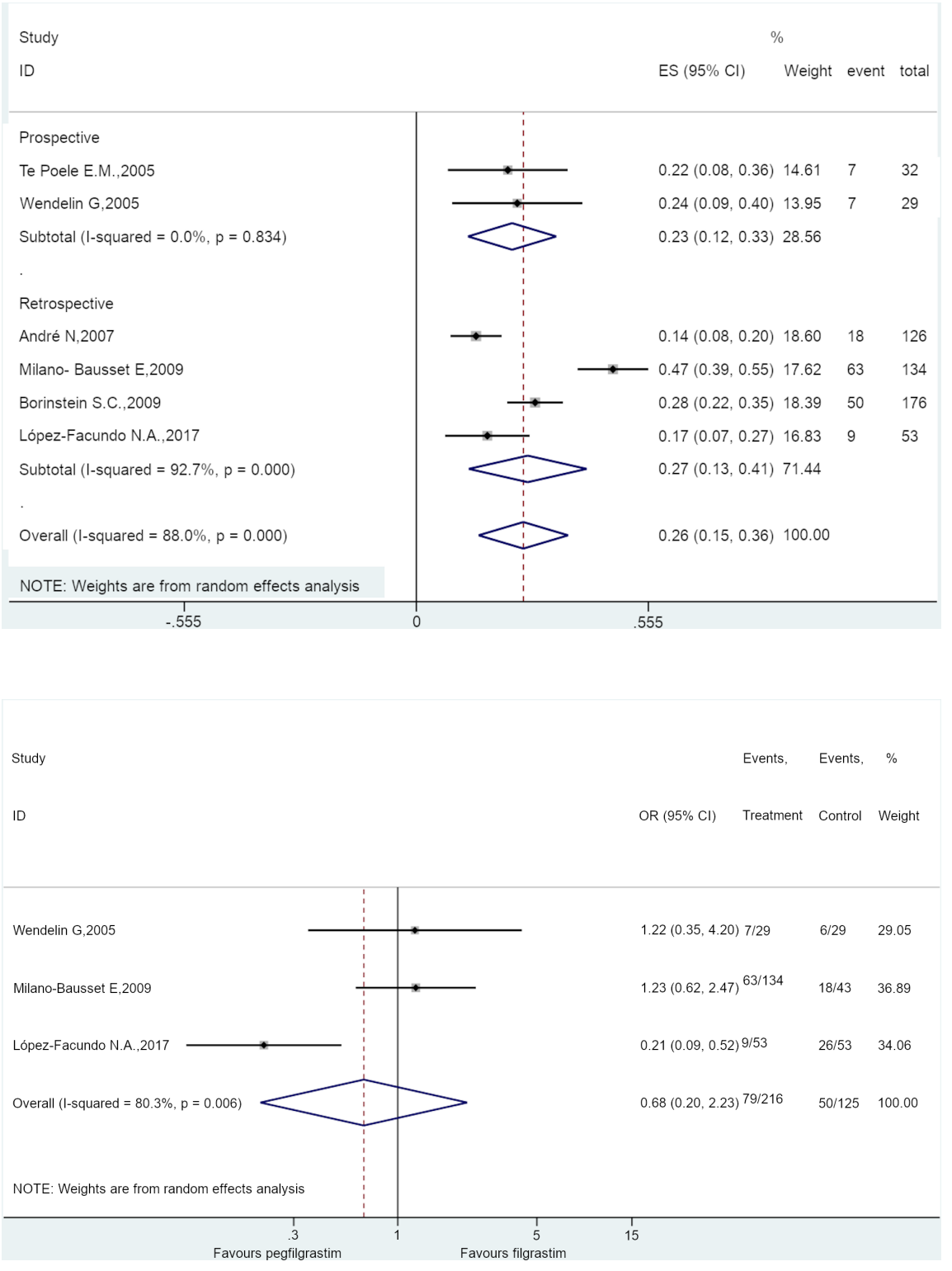




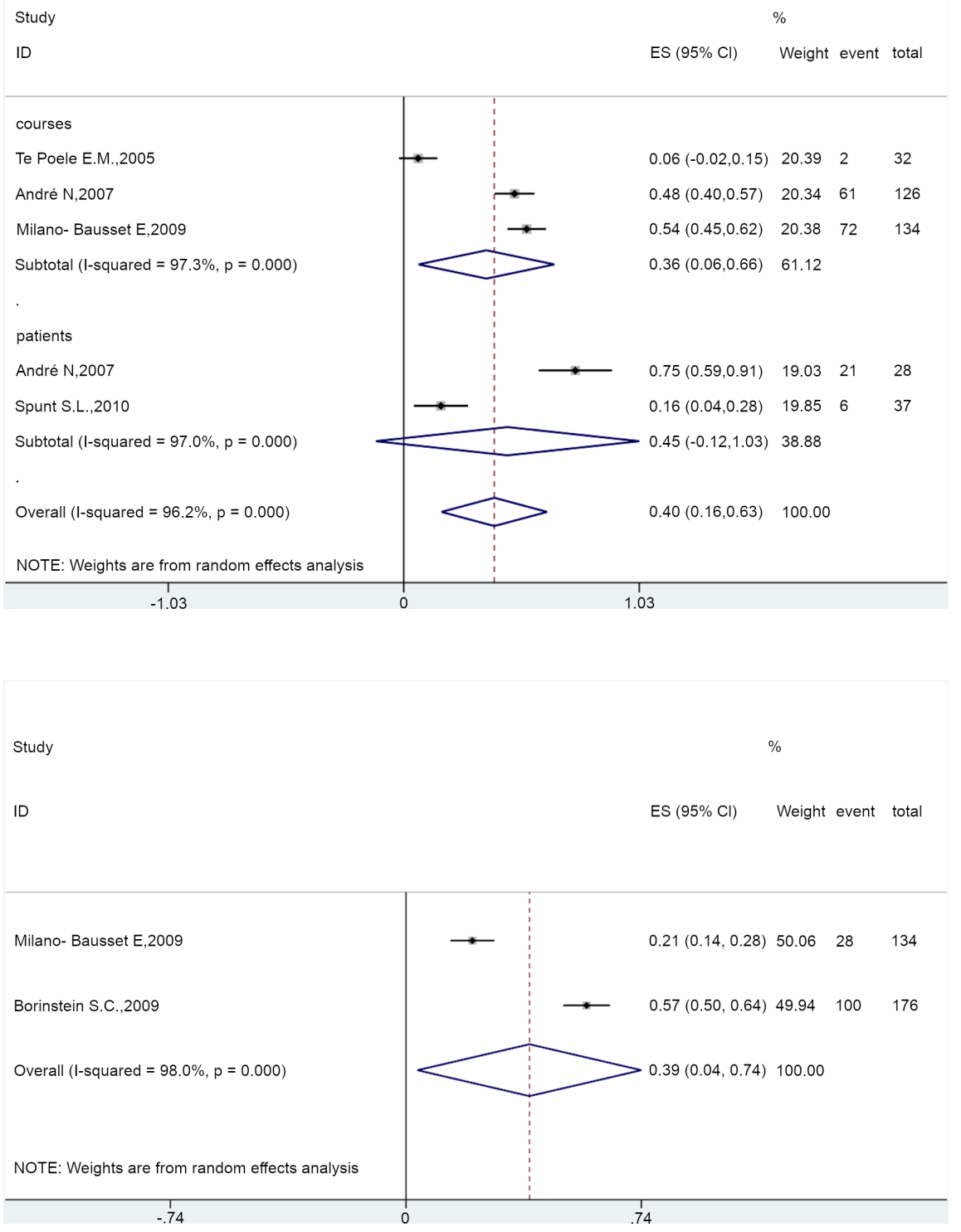


Study

ID

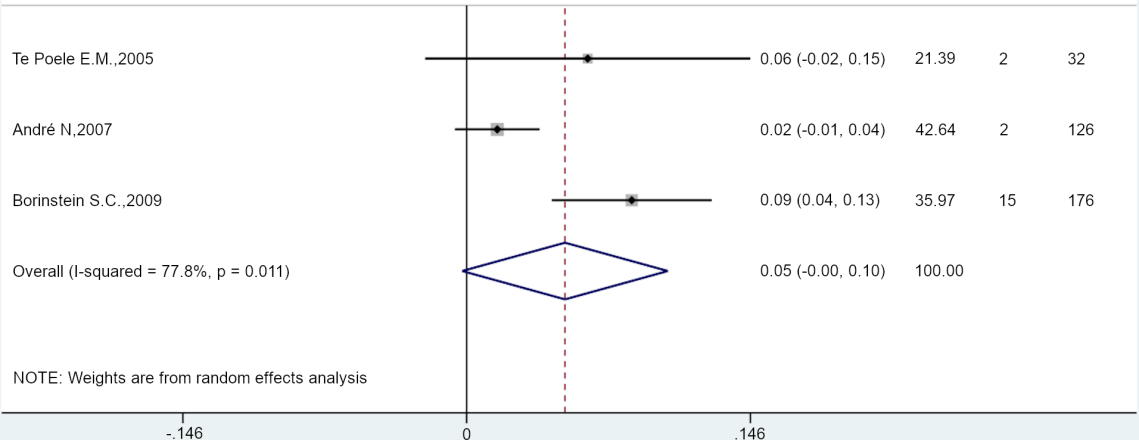

